\title{
Kommentare
}

\author{
Thomas Groß \\ Die verfassungsrechtliche Zulässigkeit gesetzlicher \\ Arbeitszeitverkürzungen in Frankreich und \\ Deutschland
}

Die Arbeitslosigkeit ist nach wie vor in den meisten Mitgliedstaaten der EU ein politisches Problem ersten Ranges, zu dessen Bekämpfung unterschiedliche Konzepte eingesetzt werden. Ein Überblick über die Lösungsansätze der Arbeitsmarktpolitik der verschiedenen Länder läßt im wesentlichen zwei Konzepte erkennen ${ }^{1}$. Zum einen haben einige Staaten wie Großbritannien und die Niederlande erhebliche Erfolge bei der Reduktion der Arbeitslosigkeit zu verzeichnen, die auf eine sehr weitgehende Deregulierung des Arbeitsmarktes zurückzuführen sind. Insbesondere ist dort die Zahl der Teilzeitbeschäftigungen deutlich erhöht worden. Diese Maßnahmen führen andererseits dazu, daß immer weniger Arbeitnehmer ihren Lebensunterhalt von dem Einkommen aus einer Stelle bestreiten können und deshalb Zweit- und Drittjobs annehmen müssen.

Frankreich hat dagegen den Weg einer staatlich vorgegebenen Verringerung der Regelarbeitszeit gewählt. Ihre Festlegung wird dem Markt und den Tarifparteien entzogen und stattdessen durch die gesetzliche Einführung der 35 -Stunden-Woche verbindlich geregelt. Dahinter steht die Einschätzung, daß der Arbeitsmarkt an einer Ungleichverteilung leidet, die zwar der Mehrzahl ein Arbeitseinkommen garantiert, eine tendenziell zunehmende Minderheit aber längerfristig oder sogar dauerhaft ausschließt. Das vorhandene Arbeitsvolumen, das sich offensichtlich nicht hinreichend vermehrt, soll deshalb durch verbindliche Vorgaben auf eine größere Zahl von Personen verteilt werden.

In Deutschland werden nach einer weitgehenden Untätigkeit in den letzten Jahren im Rahmen des korporatistischen »Bündnisses für Arbeit«verschiedene Ansätze diskutiert, vor allem der Abbau von Überstunden und die Förderung von Teilzeitbeschäftigung. Eine Intervention des Gesetzgebers zur Durchsetzung dieser Ziele ist jedoch bisher nicht absehbar, obwohl die Erfahrung zeigt, daß Arbeitszeitverkürzungen nicht in ausreichendem Umfang autonom von den Tarifparteien vereinbart werden, da sie aufgrund der Bindung an die Interessen ihrer Mitglieder keine hinreichenden Anreize zu einem Verzicht zugunsten von anderen Gruppen haben².

Alle Maßnahmen, die nicht auf freiwillige Vereinbarungen der Tarifparteien setzen, stoßen auf den Einwand, daß die verfassungsrechtliche Garantie der Tarifautonomie einer entsprechenden staatlichen Regelung entgegenstehe. In Frankreich ist eine Klage gegen das Gesetz zur Arbeitszeitverkürzung vom Verfassungsrat zurückgewiesen worden (I.). Wie eine vergleichbare Gesetzgebung in Deutschland zu beurteilen wäre, ist weniger eindeutig (II.).

I Vgl. dazu den Überblick bei Lehndorff, WSI-Mitteilungen $1998,569 \mathrm{ff}$

2 S.a. Otto, FS Zeuner, 1994, S. 12 I ffr, I 38 f. 


\section{Der Inhalt des Gesetzes}

Das französische Parlament hat mit dem Gesetz vom I 3.6. I 998 über die Verkürzung der gesetzlichen Arbeitszeit ${ }^{3}$ die Fünfunddreißig-Stunden-Woche als Regelarbeitszeit eingeführt. Die effektive gesetzliche Arbeitszeit in allen vom Arbeitsgesetzbuch erfaßten Betrieben beträgt ab dem I. I. 2000 nicht mehr neununddreißig, sondern fünfunddreißig Stunden in der Woche. Für Unternehmen mit weniger als 20 Beschäftigten gilt diese Regelung erst ab dem r. I. 2002. Die Einzelheiten zur Umsetzung dieser neuen Regelung werden in Art. 2 des Gesetzes ausdrücklich den Tarifparteien überlassen. Unternehmen, die aufgrund von tarifvertraglich vereinbarten Maßnahmen bereits vor dem gesetzlich vorgesehenen Datum die Arbeitszeit verkürzen und dadurch neue Stellen schaffen oder vorhandene erhalten, können staatliche Fördermittel erhalten, wenn bestimmte, im Gesetz festgelegte Bedingungen erfüllt sind.

Das französische Gesetz enthält sich jeder Aussage über die Folgen für die Lohnhöhe wie für die konkrete betriebliche Ausgestaltung der tatsächlichen Arbeitszeit. Es beschränkt sich auf einen punktuellen verteilungspolitischen Eingriff, dessen Folgen die Tarifparteien autonom bewältigen müssen. Ihnen bleibt hierfür ein längerer Zeitraum. Die flankierenden Regelungen haben dagegen lediglich Anreizcharakter und tangieren die Tarifautonomie nur mittelbar. Das Gesetz enthält außerdem in Art. ${ }_{3}$ einen Auftrag an die Regierung, bis spätestens 30.9.1999, d.h. noch vor Wirksamwerden der Arbeitszeitverkürzung, dem Parlament einen Bericht über seine bisherige Anwendung durch die Tarifparteien vorzulegen.

\section{Das Urteil des Verfassungsrates}

Gegen das Gesetz reichten in Frankreich mehr als sechzig Abgeordnete der Opposition Klage beim Verfassungsrat ein. Diese Form der abstrakten Normenkontrolle findet nach Art. 6r der französischen Verfassung zwischen dem Gesetzesbeschluß des Parlamentes und der Verkündung statt. Die Beschwerdeführer rügten u.a. einen Verstoß gegen die unternehmerische Freiheit (liberté d'entreprendre) und gegen das Recht auf kollektive Verhandlungen der Sozialpartner. Die unternehmerische Freiheit, die aus dem allgemeinen Freiheitsrecht des Art. 4 der Erklärung der Menschen- und Bürgerrechte abgeleitet wird, die nach wie vor geltendes französisches Verfassungsrecht ist ${ }^{4}$, sei dadurch verletzt, daß die Vertragsfreiheit der Unternehmer durch die Vorgabe eines bestimmten Ergebnissen beschnitten werde. Aus demselben Grund liege auch ein Verstoß gegen den achten Absatz der ebenfalls weitergeltenden Präambel der Verfassung von I 946 vor, der jedem Arbeiter das Recht garantiert, durch seine Delegierten an der kollektiven Festsetzung der Arbeitsbedingungen teilzunehmen.

In ihrer Stellungnahme wies die Regierung darauf hin, daß sich aus dem Gesetz allein keine Verpflichtung zu Tarifverhandlungen ergebe. Die Unternehmen seien weiterhin frei, die Arbeitszeit innerhalb der unveränderten gesetzlichen Höchstgrenzen festzusetzen. Das Prinzip der Teilnahme der Arbeiter an der kollektiven Festsetzung der Arbeitsbedingungen stehe unter einem Gesetzesvorbehalt. Diesen habe der zu Arbeitszeitregelungen berechtigte Gesetzgeber in Anspruch genommen, ohne das Ver-

3 J. O. v. I 4.6. I998, S. 9029 ; vgl. dazu auch Freyssinet, WSI-Mitteilungen 1998, $589 \mathrm{ff}$.

4 Vgl. Savoie, in: Grabitz (Hrsg.), Grundrechte in Europa und USA, Bd. I, 1986, S. 203 ff, 2 10 ff.; Colliard, Libertés publiques, 7. Aufl., 1989, S. $107 \mathrm{f}$. 
fassungsprinzip seiner Wirksamkeit zu berauben, zumal die nähere Ausgestaltung der

Arbeitszeitverkürzung durch Verhandlungen der Sozialpartner erfolge.

Der Verfassungsrat hat in seiner Entscheidung vom 10.6. 1998' das Gesetz für verfassungskonform erklärt. Er faßte dabei die Prüfung der unternehmerischen Freiheit und der Teilnahme der Arbeiter an der kollektiven Festsetzung der Arbeitsbedingungen zu einem Punkt zusammen und stützte sich dabei nur auf Art. 4 der Erklärung der Menschen- und Bürgerrechte. Er folgte der Regierung in ihrem Argument, daß die gesetzliche Regelung keinen Zwang zur kollektiven Verhandlung, sondern nur einen Anreiz enthalte. Im übrigen sah er die Regelung yon dem Gesetzesvorbehalt gedeckt, unter dem die unternehmerische Freiheit steht. Als Rechtfertigung für die Verkürzung der Arbeitszeit zog er interessanterweise den fünften Absatz der Präambel der Verfassung von 1946 heran, der jedermann das Recht zuspricht, eine Beschäftigung zu erhalten. Diesem Ziel habe der Gesetzgeber unter den gegenwärtigen Bedingungen des Arbeitsmarktes dienen wollen, ohne daß die vorgesehenen Regelungen offensichtlich ungeeignet oder unverhältnismäßig seien.

Bei der Bewertung dieses Urteils ist zu beachten, daß der französische Verfassungsrat dem Gesetzgeber generell einen weiten Einschätzungsspielraum zubilligt und sich selbst als reine Kontrollinstanz versteht ${ }^{6}$. Er hat sich deshalb jeder näheren Auseinandersetzung mit den Prognosen über die Wirkungen des Gesetzes enthalten.

\section{Arbeitszeitregelungen und Art. 9 Abs. 3 GG}

Vergleichbare Initiativen für eine gesetzliche Intervention gibt es in Deutschland zur Zeit nicht. Die starke Tradition der Tarifautonomie bildet bisher ein Bollwerk gegen solche unmittelbaren Zugriffe des Gesetzgebers auf die Arbeitszeit. Aber nicht nur eine Verkürzung der Wochenarbeitszeit, sondern auch eine arbeitsmarktpolitisch motivierte Begrenzung von Überstunden würde die Frage der Vereinbarkeit mit der Garantie der Koalitionsfreiheit in Art. 9 Abs. 3 GG aufwerfen.

\section{Die Tarifautonomie und ibre Schranken}

Eine gesetzliche Regelung der Arbeitszeit berührt die kollektive Koalitionsfreiheit, indem ein wichtiger Gegenstand von Tarifverträgen der autonomen Regelungskompetenz der Tarifparteien - zumindest teilweise - entzogen wird. Die Tarifautonomie, d. h. die rechtliche Möglichkeit, Arbeitsbedingungen durch Tarifverträge festzulegen, ist unstreitig von der kollektiven Koalitionsfreiheit umfaßt ${ }^{7}$. Eine eindeutige Bestimmung des möglichen Regelungsinhaltes ist allerdings nicht möglich, da der Sachbereich sich verändert und deshalb auch der Schutzbereich der Koalitionsfreiheit entwicklungsoffen verstanden werden muss ${ }^{8}$. Es ist aber unstrittig, daß die Regelung der Arbeitszeit zu den Arbeitsbedingungen zu zählen ist, die zum tarifvertraglichen Regelungsbereich gehören. Das Grundrecht sichert die Rechtsmacht eines Zusammenschlusses von Individuen, insbesondere der Arbeitnehmer, aber auch der Arbeit-

5 J.O. v. 14.6.1998, S. 9033 .

6 Roussillon, Le Conseil constitutionnel, 1991, S. $68 \mathrm{ff}$

7 BVerfGE 44, 322, 344; 50, 290, 367; 92, 26, 38; $94,268,283$; Dreier-Bauer, GG, Bd. I, 1996, Art. 9 Rn. 78 ; Jarass/Pieroth, GG, 4. Aufl., 1997, Art. 9 Rn. 29; Löwisch, in: Richardi/Wlotzke (Hrsg.), Munchener Handbuch zum Arbeitsrecht, Bd. 3, 1993, $\$ 239$ Rn. 40.

8 Scholz, HStR VI, 1989, 1 ISI Rn. 16; Rupp, JZ 1998, 919 ff., 924 f; zur Auslegung s. Waitermann, NZA I991, 754 ff., 757 ff.; Meik, Der Kernbereich der Tarifautonomie, 1 987, S. 73 ff.

9 BVerfGE 94, 268, 283; Dreier-Bauer, GG, Bd. 1, Art. 9 Rn. 70; Jarass/Pieroth, GG, Art. 9 Rn. 23; SachsHofling, GG, 2. Aufl., 1999, Art. 9 Rn. 54; Kemper, Die Bestimmung des Schutzbereiches der Koalitionsfreiheit (Art. 9 Abs. 3 GG), r 990, S. 123; Sacker/Oetker, Grundlagen und Grenzen der Tarifautonomie, $199^{2}$, S. 87 . 
geber, durch Vertragsschluß für alle ihre Mitglieder verbindliche Normen im Bereich der Arbeits- und Wirtschaftsbedingungen festzulegen. Letztlich sind alle individuellen Aspekte der Koalitionsfreiheit und die übrigen Elemente der kollektiven Betätigungsfreiheit auf diese Normsetzungsbefugnis ausgerichtet.

Die ältere Rechtsprechung des Bundesverfassungsgerichts ließ die grundrechtsdogmatische Einordnung gesetzlicher Regelungsbefugnisse durch die Bezugnahme auf einen Kernbereich koalitionsmäßiger Betätigung, der keinen inhaltlich unbeschränkten und gesetzlich unbeschränkbaren Handlungsspielraum der Tarifparteien einräu$\mathrm{me}^{\mathrm{io}}$, im unklaren. In jüngerer Zeit wird dagegen zutreffend zwischen der gesetzlichen Ausgestaltung der Koalitionsfreiheit und ihrer Beschränkung unterschieden ${ }^{11}$. Eine Ausgestaltungsbefugnis des Gesetzgebers, die gleichzeitig eine Ausgestaltungspflicht ist, besteht insoweit, als sie zur Verwirklichung der Koalitionsfreiheit unabdingbar ist. Hierzu gehört als Kernstück der Betätigungsfreiheit der Koalitionen die Tarifautonomie, d. h. die grundsätzliche Anerkennung der autonomen Regelungsbefugnis der Tarifparteien, wie sie durch das Tarifvertragsgesetz erfolgt ist ${ }^{12}$. Der Gesetzgeber muß ein rechtliches Instrumentarium zur Verfügung stellen, das solche kollektive Bindungen ermöglicht, wie sie durch den $Z$ weck der Koalitionsfreiheit als Chance realer Vertragsfreiheit durch Zusammenschluss gefordert wird ${ }^{13}$. Einen Gestaltungsspielraum hat der Gesetzgeber nicht bei der Frage, ob ein Tarifvertragssystem bereitgestellt wird, sondern nur bei der näheren Bestimmung der Gegenstände eines Tarifvertrags.

Inhaltliche Regelungen im Bereich der Arbeits- und Wirtschaftsbedingungen, die in den Bereich fallen, der den Tarifparteien zur autonomen Regelung zugewiesen ist, sind dagegen in jedem Fall Beschränkungen der Koalitionsfreiheit, d.h. ihrer durch Art. 9 Abs. 3 GG geschützten Regelungsbefugnis. Eine Berufung auf den Volkssouverän, der nicht ausgeschaltet werden könne ${ }^{14}$, ist hierbei verfehlt, denn für Art. 9 Abs. 3 GG gilt selbstverständlich ebenfalls die Aussage des Art. 1 Abs. 3 GG, daß auch der Gesetzgeber an die Grundrechte gebunden ist. Gesetzgeberische Beschränkungen des Handlungsspielraums der Koalitionen sind nichtsdestotrotz möglich, sie müssen sich jedoch an die allgemeinen grundrechtsdogmatischen Voraussetzungen halten. Mangels ausdrücklicher Schrankenvorbehalte bestehen folglich nur verfassungsimmanente Schranken, die beschränkende Regelung muß dem Schutz eines in der Verfassung verbürgten Rechtsgutes dienen ${ }^{15}$. Der von einigen Stimmen in der Literatur postulierte unspezifische Gemeinwohlvorbehalt der Koalitionsfreiheit ${ }^{16}$ findet dagegen weder im Wortlaut des Grundgesetzes noch in den allgemeinen Grundrechtslehren eine Basis ${ }^{17}$. Die Maßstäbe für die Zulässigkeit von Grundrechtseinschränkungen können nicht einem abstrakten Begriff entnommen werden, sondern bedürfen konkreter Anknüpfungspunkte in der Verfassung.

\footnotetext{
10 BVerfGE 38, 386, 393; 44, 322, 24 I f.; 50, 290, 368; krit. Farthmann/Coen, HbdVerfR, 2. Aufl., I994, $\$$ I9 Rn. $30 \mathrm{ff}$.

II BVerfGE 93, 352, 358ff. m.w.N.

12 AK-GG-Kittner, 2. Aufl., 1989, Art. 9 III Rn. 65; Farthmann/Coen, HbdVerfR, $\mathbb{1} 19$ Rn. 62; Biedenkopf, Grenzen der Tarifautonomie, 1964, S. 104 f.

I3 AK-GG-Kittner, Art. 9 III Rn. 24; Otto, FS Zeuner, S. I 2 I ff., I 3 I.

I4 V. Münch/Kunig-Lower, GG, 4. Aufl., I992, Art. 9 Rn. 7I (Tarifautonomie); so wohl auch Lowisch, in: Richardi/Wlotzke (Hrsg.), Münchener Handbuch zum Arbeitsrecht, Bd. 3, $\$ 239$ Rn. 48.

is BVerfGE 92, 26, 41; Schwerdtfeger, in: Max-Planck-Institut für auslandisches offentliches Recht und Völkerrecht, Die Koalitionsfreiheit des Arbeitnehmers, 1980, Bd. I, S. 149 ff., 217 ; Hagemeister/Kempen/ Zachert/Zilius, TVG, 2. Aufl., 1990, Einleitung, Rn. 106; Otto, FS Zeuner, S. 121 ff., 137 f.; in BVerfGE 94, 268, 284, wird diese Frage offengelassen; zu weiteren Schrankenkonstruktionen vgl. Knebel, Koalitionsfreiheit und Gemeinwohl, 1978, S. 64 ff.

I6 Z. B. Scholz, HStR VI, \$IS I Rn. 3 I ff.; Säcker, Grundprobleme der kollektiven Koalitionsfreiheit, 1969, S. sı ff.; hierzu ausfuhrlich Knebel, Koalitionsfreiheit und Gemeinwohl, 1978, S. $78 \mathrm{ff}$

17 Krit. auch v. Münch/Kunig-Lower, GG, 4. Aufl. Art. 9 Rn. 58; Pieroth/Schlink, Grundrechte, Rn. 758.
} 
Als eine solche Grundlage für Einschränkungen der Tarifautonomie kommt in diesem

Zusammenhang das Sozialstaatsprinzip in Frage. Während sich das bisherige Arbeitszeitrecht mit seinen Mindestbedingungen gemäß $\$$ I AZG auf den Schutz der Gesundheit der Arbeitnehmer und damit auf Art. 2 Abs. 2 S. I GG stützt ${ }^{18}$, könnte sich eine arbeitsmarktpolitisch motivierte gesetzliche Verkürzung nicht mehr auf dieses Grundrecht berufen, sondern müßte auf das soziale Staatsziel zurückgreifen. Es ist auch bereits bisher als Grundlage für verschiedene gesetzliche Regelungen im Arbeitsrecht angesehen worden, die in der Regel Mindeststandards festlegen, die dadurch der Dispositionsfreiheit der Tarifparteien entzogen $\operatorname{sind}^{19}{ }^{19}$. Es gibt jedoch keine erkennbaren Gründe, warum das Sozialstaatsprinzip auf die Festlegung von Mindeststandards beschränkt werden müßte ${ }^{20}$. Es verlangt vielmehr einen sozialen Ausgleich, der über die Sicherung des Existenzminimums hinausgeht ${ }^{2 r}$. Die dauerhafte Massenarbeitslosigkeit stellt aber zweifellos ein soziales Problem ersten Ranges dar, das von keiner wichtigen politischen Kraft als hinzunehmendes Übel angesehen wird. Auch wenn - jedenfalls auf Bundesebene ${ }^{22}$ - eine explizite Verbürgung eines Rechtes auf Arbeit fehlt, kann man dem Gesetzgeber nicht von vornherein das Recht absprechen, neue Wege zur Reduktion der Arbeitslosigkeit zu beschreiten.

\section{Grenzen der Beschränkung der Koalitionsfreibeit}

Wenn man das Sozialstaatsprinzip als eine ausreichende Grundlage ansieht, ist zu klären, ob durch eine gesetzliche Festlegung der Regelarbeitszeit in den Kernbereich der Tarifautonomie eingegriffen würde, der gesetzlichen Beschränkungen entzogen ist. Diese Frage stößt aufgrund der Rechtsgeprägtheit des Grundrechtes auf besondere Schwierigkeiten. Entsprechend der Unterscheidung zwischen Ausgestaltung und Beschränkung kann man die Frage nach dem Kernbereich nicht auf den Tarifvertrag als Handlungsinstrument beschränken, sondern muß nun nach materiellen Grenzen gesetzlicher Regelungen fragen. Art. 9 Abs. 3 GG verlangt, daß der Zuständigkeitsbereich der Tarifparteien nicht ausgehöhlt wird ${ }^{23}$. In der Literatur wird v. a. die gesetzliche Lohnkorrektur als Eingriff in den Wesensgehalt angesehen ${ }^{24}, \mathrm{z}$. T. aber auch die Regelung der Arbeitszeit ${ }^{25}$. Andere nehmen dagegen an, daß es nicht möglich sei, einen Kernbereich der Tarifautonomie abstrakt zu bestimmen ${ }^{26}$. Es müsse lediglich gewährleistet sein, daß den Tarifparteien per saldo ein ausreichend großes Feld autonomer Regelungsmöglichkeiten verbleibe. Es könne jedoch kein Regelungsgegenstand von vornherein dem Zugriff des Gesetzgebers entzogen werden.

I 8 Vgl. dazu Schliemann/Forster/Meyer, Arbeitszeitrecht, 1997; zur historischen Entwicklung Muckenberger, in: Offe/Hınrichs/Wiesenthal (Hrsg.), Arbeitszeitpolitik, I982, S. $46 \mathrm{ff}$.

19 Schwerdtfeger, in: Max-Planck-Institut für ausländisches offentliches Recht und Volkerrecht, Die Koalitionsfreiheit des Arbeitnehmers, 1980, Bd. I, S. 149 ff., 166 f.; s. a. Sachs-Höfling, GG, Art. 9 Rn. 135 ; Dreier-Bauer, GG, Bd. 1, Art. 9 Rn. 94 Fn. 456.

20 Vgl. Dreier-Gröschner, GG, Bd. 2, 1998, Art. 20 (Sozialstaat) Rn. 28; s. a. Merli, in: Hofmann/Holländer/ Merli/Wiederin (Hrsg.), Armut und Verfassung, 1998, S. $117 \mathrm{ff} ., 122$.

21 Kleindiek, in: Erberich/Hörster/Hoffmann/Kingreen/Punder/Stormer(Hrsg.), Frieden und Recht, 1998, S. II ff., $127 \mathrm{ff}$

22 Vgl. aber die Landesyerfassungen von Bayern (Art. 166 II), Berlin (Art. 12 I), Brandenburg (Art. 48 I), Hessen (Art. $28 \mathrm{II}$ ), Nordrhein-Westfalen (Art. 24 I 3), Rheinland-Pfalz (Art. 53 II) und Saarland (Art. 45 ).

23 Biedenkopf, Grenzen der Tarifautonome, S. 104, I 53 ; Kemper, Die Bestimmung des Schutzbereiches der Koalitionsfreiheit (Art. 9 Abs. 3 GG), S. 121 .

24 V. Munch/Kunig-Lower, GG, 4. Aufl. Art. 9 Rn. 7 I (Tarifautonomie); AK-GG-Kittner, Art. 9 III Rn. 65 ; Meik, Der Kernbereich der Tarifautonomie, S. I 32; a.A. Lowisch, in: Richardi/Wlotzke (Hrsg.), Munchener Handbuch zum Arbeitsrecht, Bd. 3, \$239 Rn. 49; Knebel, Koalitionsfreiheit und Gemeinwohl, S. I $66 \mathrm{ff}$., hält einen staatlichen Genehmigungsvorbehalt für zulässig.

2s Ehmann, Bitburger Gesprache 198 s, S. 19 ff., 33.

26 Kemper, Die Bestimmung des Schutzbereiches der Koalitionsfreiheit (Art. 9 Abs. 3 GG), S. I22; Lowisch, in: Richardi/Wlotzke (Hrsg.), Münchener Handbuch zum Arbeitsrecht, Bd. 3, \$239 Rn. 4 . 
An dieser Stelle drängt sich eine Parallele zur Diskussion um die verfassungsrechtliche Gewährleistung der kommunalen Selbstverwaltung auf. Das durch Art. 28 Abs. 2 GG geschützte Selbstverwaltungsrecht der Gemeinden umfaßt ihre Rechtsetzungsautonomie ${ }^{27}$, jedoch läßt sich nach der Rechtsprechung des Bundesverfassungsgerichtes kein gegenständlicher Kernbereich fixieren ${ }^{28}$. Zwar handelt es sich hierbei um ein Frage aus dem Bereich der Staatsorganisation, im anderen Fall um eine besondere Form der Privatautonomie, doch ist Art. 28 Abs. 2 GG ein grundrechtsgleiches Recht mit vergleichbarer Struktur. Wie bei der Koalitionsfreiheit des Art. 9 Abs. 3 GG ist der Schutzbereich normkonstituiert. In beiden Fällen ist zwischen der Garantie des Instrumentes der Normsetzungsmacht und der fehlenden Bestimmbarkeit eines Kerns von unentziehbaren Regelungsgegenständen zu unterscheiden. Folglich käme es bei jeder gesetzlichen Festsetzung der Arbeitszeit auf fünfunddreißig Wochenstunden darauf an, welcher Spielraum den Tarifparteien im übrigen noch verbleibt.

Ein weiterer Punkt, an dem Bedenken gegen eine sozialstaatlich begründete Arbeitszeitverkürzung geltend gemacht werden können, ist die Verhältnismäßigkeit der Regelung. Ein großes Problem wird schon die Geeignetheit darstellen. Eine Prognose, ob sich auf diesem Weg quantitativ relevante Umverteilungseffekte erzielen lassen, stößt auf komplexe makroökonomische Zusammenhänge, zumal angesichts zunehmender internationaler Verflechtungen ${ }^{29}$. Einerseits muß jedoch für diese Frage wie für andere wirtschaftspolitische Entscheidungen dem Parlament ein besonders weiter Einschätzungsspielraum eingeräumt werden ${ }^{30}$. Andererseits kann zumindest auf einige Untersuchungen über die Wirkungen der bisherigen tarifvertraglich vereinbarten Arbeitszeitverkürzungen zurückgegriffen werden, die einen quantitativ nachweisbaren Effekt belegen, auch wenn er unter den rein rechnerisch ermittelten Werten liegt und stark von den Begleitumständen abhängig ist ${ }^{3{ }^{3}}$. Im übrigen bliebe der Gesetzgeber verpflichtet, den Einfluß der Regelung auf die Entwicklung am Arbeitsmarkt zu beobachten und gegebenenfalls korrigierend einzugreifen ${ }^{32}$.

Für die Frage der Erforderlichkeit gilt ebenfalls der Hinweis auf die gesetzgeberische Einschätzungsprärogative. Es gab in den letzten Jahrzehnten bereits eine Fülle von unterschiedlichsten Steuerungsversuchen durch fiskalische Maßnahmen, Subventionen, Deregulierung und andere Anreizsysteme, ohne daß man ihnen einen durchschlagenden Erfolg bescheinigen könnte. Der Nachweis, daß andere, mildere Mittel besser geeignet wären, das angestrebte Ziel einer Entlastung des Arbeitsmarktes zu erreichen, dürfte nicht zu führen sein.

Eine schwierige Frage bleibt die Angemessenheit, die Verhältnismäßigkeit im engeren Sinn. Das Bundesverfassungsgericht geht davon aus, daß die Wirkkraft des Grundrechtes aus Art. 9 Abs. 3 GG in dem Maße zunimmt, in dem die Tarifparteien die Materie aus Sachgründen angemessener regeln können und knüpft dabei an die Praxis der Tarifverträge $a^{33}$. Nach diesem Kriterium sind Arbeitszeitregelungen besonders heikel. Allerdings ist die Frage der Angemessenheit der bisherigen tarifvertraglichen

\footnotetext{
27 Dreier, GG, Bd. 2, Art. 28 Rn. 133.

28 BVerfGE $79,127,146$.

29 Vgl. z. B. Kühl, in: Mayr/Janßen (Hrsg.), Perspektiven der Arbeitszeitverkuirzung, I984, S. 100ff.; Bolle, in: Kutsch/Vilmar (Hrsg.), Arbeitszeitverkurzung, 1983, S. 19r ff.; Renner, Arbeitszeitverkurzung als beschaftigungspolitisches Instrument, Diss. Hohenheim, $1983, \mathrm{~S} .92 \mathrm{ff}$.

30 Dreier-Wieland, GG, Art. I 2 Rn. 129 ff. mit Nachweisen.

3 I Seifert, WSI-Mitteilungen I998, 579 ff., 58 I f.; Bosch, WSI-Mitteilungen 1998, 345 ff., 35 I ff.; Stille/Zwiener, Arbeits- und Betriebszeiten in Deutschland, DIW-Sonderheft 160,1997, S. $83 \mathrm{ff}$

32 Vgl. zur Nachbesserungspflicht des Gesetzgebers BVerfGE 50, 290, 335; 88, 203, $309 \mathrm{f}$

33 BVerfGE 94, 268, $284 \mathrm{f}$.
} 
Regelungen angesichts der fortdauernden Massenarbeitslosigkeit gerade fraglich. Wie die Abwägung zwischen dem sozialstaatlichen Ziel der gleichmäßigeren Verteilung von Arbeitseinkommen und dem Schutz der Regelungsmacht der Tarifparteien zu erfolgen hat, würde entscheidend von der konkreten Ausgestaltung eines entsprechenden Gesetzes abhängen.

\title{
Fredrik Roggan /Jan Sürig Aufenthaltsverbot für Drogenabhängige
}

\author{
Aufenthaltsverbot für Drogenhändler \\ I. Eine Platzverweisung und ein längerfristiges Aufenthaltsverbot stellen qualitativ \\ unterschiedliche polizeiliche Maßnabmen dar. Sie bezieben sich auf nach Art und \\ Ausmaß nicht vergleichbare Gefabrenlagen. Rechtsgrundlage für eine Platzverwei- \\ sung bildet $\ I_{4}$ BremPolG, für ein längerfristiges Aufentbaltsverbot die polizeiliche \\ Generalklausel in $\$ 10$ Abs. I BremPolG. \\ 2. Ein längerfristiges Aufenthaltsverbot berübrt den Schutzbereich von Art. II \\ Abs. I GG. Einschränkungen des Grundrechts sind - unter Beachtung der in \\ Art. II Abs. 2 GG genannten Schranken - auch im Rabmen des landesrechtlichen \\ Gefabrenabwebrrechts zulässig. \\ 3. Die Bekämpfung des Drogenbandels und -konsums in der sogenannten offenen \\ Drogenszene kann ein längerfristiges Aufenthaltsverbot rechtfertigen. Aus Gründen \\ der Verbältnismäßigkeit darf ein solches Verbot nur gegen Personen ergehen, die in \\ besonderer Weise an der Bildung und Aufrechterhaltung der Szene beteiligt sind.
}

OVG Bremen, U.v. 24.3. 1998, OVG I BA 27/97, 2 A 149/96.

\section{Die Entscheidung}

In diversen Großstädten gibt es in den letzten Jahren Versuche, den Aufenthalt von Angehörigen sozialer Randgruppen im Innenstadtbereich zu reglementieren. Z.T. betrifft dies das Betteln, teils den öffentlichen Alkoholkonsum, teils - so das obige Bremer Beispiel - Angehörige der sog. offenen Drogenszene, die häufig durch Kleinkriminalität aufgefallen sind. Mit »offener Drogenszene» sind diejenigen Treffpunkte gemeint, an denen Drogenabhängige illegalisierte Drogen nur oberflächlich verdeckt konsumieren und/oder damit handeln. In dem Fall, den das OVG Bremen zu entscheiden hatte, war von der Ordnungsbehörde (Stadtamt) ein 6-monatiges Aufenthaltsverbot für ein mehrere Quadratkilometer großes innenstadtnahes Gebiet ausgesprochen worden ${ }^{\text {. }}$. In den Aufenthaltsverboten liegt somit auch ein wesentliches Moment einer Verdrängungspolitik in den Innenstädten durch polizeiliche Maßnahmen.

Das Urteil des OVG Bremen hat neben diesem Aspekt aber auch eine große Bedeutung für die polizeirechtliche Systematik. Es betrifft die Frage, ob ein Aufenthaltsverbot auf die polizeiliche Generalklausel gestützt werden kann. Diese Frage stellt sich deshalb, weil das Bremer Polizeigesetz (BremPolG) - anders als etwa das von Niedersachsen ( $\$$ I 7 Abs. 2 NGefAG) - keine Spezialermächtigung enthält, auf die sich längerfristige und weiträumige Aufenthaltsverbote stützen lassen.

I OVG Bremen, NVwZ I999, 3 is. 